\title{
FUBINI THEOREMS FOR ORLICZ SPACES OF LEBESGUE-BOCHNER MEASURABLE FUNCTIONS
}

\author{
VERNON ZANDER ${ }^{1}$
}

\begin{abstract}
Let $(X, V, v)$ be the volume space formed as the product of the volume spaces $\left(X_{i}, V_{i}, v_{i}\right)(i=1,2)$. Let $(p, q)$ be a pair of complementary (continuous) Young's functions, let $Y, Z$, $Z_{1}, Z_{2}, W$ be Banach spaces, let $w$ be a multilinear continuous operator on $Y \times Z_{1} \times Z_{2} \rightarrow W$. Let $L_{p}(v, Y)$ be the Orlicz space of Lebesgue-Bochner measurable functions generated by $p$, and let $K_{q}(v, Z)$ be the associated space of finitely additive $Z$-valued set functions. The principal result of this paper is as follows: Let $f \in L_{p}(v, Y), \mu_{2} \in K_{q}\left(v_{2}, Z_{2}\right)$. Then (a) the function $f\left(x_{1}, \cdot\right)$ is $v_{2}$ Orlicz summable $v_{1}$-a.e.; (b) the operator $r\left(f, \mu_{2}\right)$ defined by the expression $r\left(f, \mu_{2}\right)\left(x_{1}\right)=\int w_{1}\left(f\left(x_{1}, x_{2}\right), \mu_{2}\left(d x_{2}\right)\right) v_{1}$-a.e. is bilinear and continuous from $L_{p}(v, Y) \times K_{q}\left(v_{2}, Z_{2}\right)$ into $L_{p}\left(v_{1}, Y_{1}\right) / N$, where $w_{1}\left(y, z_{2}\right)=w\left(y, z_{2}\right)$, where $Y_{1}$ is the Banach space of bounded linear operators from $Z_{1}$ into $W$, and where $N$ is the set of $Y_{1}$ valued $v_{1}$-measurable functions of zero seminorm; (c) the equality $\int w\left(f, d \mu_{1}, d \mu_{2}\right)=\int w_{0}\left(r\left(f, \mu_{2}\right), d \mu_{1}\right)$ holds for all $f \in L_{p}(v, Y)$, $u_{i} \in K_{q}\left(v_{i}, Z_{i}\right)(i=1,2)$, where $w_{0}\left(y_{1}, z_{1}\right)=y_{1}\left(z_{1}\right)$ for all $y_{1} \in Y_{1}$, $z_{1} \in Z_{1}$.
\end{abstract}

The purpose of this paper is to complete the basic integration theory for the Orlicz spaces of Bochner measurable functions. In establishing the initial part of this theory of Orlicz spaces, Diestel [4] established the following results: a characterization of convergence, a monotone convergence theorem, a dominated convergence theorem, and the density of simple functions in the Orlicz space. The Fubini type results of this paper essentially complete the basic theory of Orlicz spaces of Bochner summable functions.

Presented to the Society, August 27, 1970; received by the editors March 20, 1970 and, in revised form, February 1, 1971.

AMS 1970 subject classifications. Primary 28A35, 28A40, 46E30; Secondary 28A25, 28A45, 46G10, 46E40.

Key words and phrases. Fubini theorem, Orlicz spaces, finitely additive set functions, Lebesgue-Bochner measurable functions.

${ }^{1}$ This paper is based largely upon portions of the author's doctoral dissertation which was written under the direction of Professor W. M. Bogdanowicz. The research was supported in part by the National Aeronautics and Space Administration and preparation of the paper was supported in part by West Georgia Faculty Research Grant No. 7028.

(c) American Mathematical Society 1972 
The Orlicz spaces treated in this paper deviate from the classical Orlicz spaces in that, instead of their being generated from the usual measure space, we shall generate them from a volume space and from the ensuing integration theory of Bogdanowicz [1]. The volume space is a natural environment for Fubini type theorems. That it is also a valid environment is established by the work of Diestel [4] and by the extensive work of Bogdanowicz (see [3] for a suitable bibliography). The notions of volume and prering are not alien to integration theory, since a family of measurable sets on which a measure is finite is a prering and the measure restricted to this domain is a volume.

1. Preliminaries. This paper will follow the notation of Diestel [4]. The principal deviation from standard Orlicz space notation is that we will use the letters $p, q$ for the complementary Young's functions. Abbreviations of the form Th. 1, Th. 3 and so on, will refer to the corresponding results in reference [4]. The underlying integration theory we shall assume in this paper is the theory developed by W. Bogdanowicz [1].

Let $V$ be a family of subsets of an abstract set $X$ such that for any two sets $A, B$ from $V$ we have $A \cap B \in V$ and $A-B$ is a finite union of disjoint sets from $V$. The family $V$ is called a prering.

A nonnegative, real-valued function $v$ defined on the prering $V$ is called a volume if for every countable family of disjoint sets $A_{t} \in V(t \in T)$ such that $A=\bigcup_{t \in T} A_{t} \in V$ we have $v(A)=\sum_{T} v\left(A_{t}\right)$.

The triple $(X, V, v)$ is called a volume space. For $Y$ a Banach space the space $S(V, Y)$ of $V$-simple $Y$-valued functions and the space $L(v, Y)$ of Lebesgue-Bochner summable functions are defined in Bogdanowicz [1], as are the notions of $v$-null sets and $v$-a.e. The space $M(v, Y)$ of $Y$-valued $v$-measurable functions is defined in Bogdanowicz [2].

Let $(p, q)$ be a pair of nonegative, real-valued functions defined on the interval $[0, \infty)$ such that $p$ is continuous from $(0, \infty)$ onto $(0, \infty)$ and differentiable with derivative $p^{\prime}$ on $(0, \infty), p^{\prime}$ is a homeomorphism of $(0, \infty)$ with itself such that if $\left(p^{\prime}\right)^{-1}$ denotes the inverse function of $p^{\prime}$ then

$$
q(x)=\int_{0}^{x}\left(p^{\prime}\right)^{-1}(t) d t
$$

Then $(p, q)$ are called complementary Young's functions.

We say that the function $p$ satisfies the $\Delta_{2}$-condition if there exists a constant $M>0$ such that $p(2 x) \leqq M p(x)$ for all nonnegative $x$. We say that the function $p$ satisfies the $\Delta^{\prime}$-condition if there exists a constant $k>0$ such that $p(x y) \leqq k p(x) p(y)$ for all nonnegative $x$ and $y$.

Let $Y, Z, W$ be Banach spaces and let $U$ be the space of all bilinear continuous operators on the space $Y \times Z$ into the space $W$. Denote the 
norms of the above spaces by $\mid \cdot$. Assume that $u \in U$. Let $(X, V, v)$ be a volume space and let $(p, q)$ be a pair of complementary Young's functions.

Let $L_{p}(v, Y)$ be the space of functions $f$ mapping the space $X$ into the space $Y$ which satisfy the following conditions:

(i) $f$ is $v$-locally summable (that is, $c_{A} f \in L(v, Y)$ for all $A \in V$ );

(ii) $f$ has a support from $V_{\sigma}$ (that is, there exist $A_{i} \in V, i=1,2, \cdots$, such that $f(x)=0$ if $x \notin \bigcup_{i} A_{i}$ );

(iii) $\|f\|_{p, v}<\infty$, where the functional $\|f\|_{p, v}$ is defined by the formula

$$
\|f\|_{p, v}=\sup \left\{\int|f| s d v: s \in Q\left(v, R^{+}\right)\right\}
$$

where $Q\left(v, R^{+}\right)$, which shall be called the norming set for the space $L_{p}(v, Y)$, is the set of all nonnegative $V$-simple functions $s$ such that $\int q \circ s d v \leqq 1$.

According to Th. 2 the space $\left(L_{p}(v, Y),\|\cdot\|_{p, v}\right)$ is a complete seminormed linear space. If it is assumed that the Young's function $p$ satisfies the $\Delta_{2}$-condition then the space $S(V, Y)$ of simple functions is dense in the space $L_{p}(v, Y)$. If we define the functional $\|\cdot \cdot\|_{p}$ on the space $L_{p}(v, Y)$ by the expression

$$
f \|_{p}=\inf \left\{k>0: \int p(f / k) d v \leqq 1\right\}
$$

then it follows easily from Young's inequality and from Th. 4 that $\|\cdot\|_{p}$ is a seminorm on $L_{p}(v, Y)$ which is equivalent to the seminorm $\|\cdot\|_{p, v}$.

2. Spaces of finitely additive set functions. In this section we shall discuss two spaces of finitely additive set functions which are related to the Orlicz space $L_{p}(v, Y)$, one space of which has two equivalent formulations.

For $Z$ a Banach space and $u \in U$ let $K_{q, u}(v, Z)$ be the space of all finitely additive functions $\mu$ mapping the prering $V$ into the Banach space $Z$ such that $\mu(A)=0$ if $v(A)=0$ and the functional $\|\mu\|_{\boldsymbol{Q}, u}$ is finite, where

$$
\begin{aligned}
\|\mu\|_{q, u}=\sup \left\{\left|\sum_{i} u\left(y_{i}, \mu\left(A_{i}\right)\right)\right|: y_{i} \in Y,\left\{A_{i}\right\}\right. \text { is a finite family } \\
\left.\quad \text { of disjoint sets from } V \text { and }\left\|\sum_{i} y_{i} c_{A_{i}}\right\| p, v \leqq 1\right\} .
\end{aligned}
$$

It is clear that the space $K_{q, u}(v, Z)$ is a seminormed space.

Let $K_{q}(v, Z)$ be the space of finitely additive functions $\mu$ mapping the prering $V$ into the Banach space $Z$ such that $\mu(A)=0$ if $v(A)=0$ and the 
functional $\|\mu\|_{q}$ is finite, where

$$
\begin{aligned}
\|\mu\|_{q}=\sup \left\{\sum_{i} a_{i}\left|\mu\left(A_{i}\right)\right|: a_{i} \geqq 0,\left\{A_{i}\right\}\right. \text { is a finite family } \\
\\
\text { of disjoint sets from } \left.V \text { and }\left\|\sum_{i} a_{i} c_{A_{i}}\right\|_{p, v} \leqq 1\right\} .
\end{aligned}
$$

We notice that the space $K_{q}(v, Z)$ is a subset of the space $K_{q, u}(v, Z)$ and $\|\mu\|_{q, u} \leqq|u|\|\mu\|_{q}$ for all $\mu \in K_{q}(v, Z)$.

Denote by $A^{q}(v, Z)$ the space of all finitely additive functions $\mu$ from the prering $V$ into the space $Z$ which satisfy the following conditions: (i) $\mu(A)=0$ when $v(A)=0$, for $A \in V$, (ii) $I_{q}(\mu / k) \leqq 1$ for some positive $k$, where the function $I_{q}$ is defined by the following expression:

$$
I_{q}(\mu)=\sup \sum\{q(|\mu(A)| / v(A)) v(A): A \in \mathscr{F}\}
$$

where the supremum is taken over all finite families $\mathscr{F}$ of disjoint sets from the prering $V$ (we observe the convention $0 / 0=0$ here and throughout). By Theorem 11 of Uhl [7] the functional $N_{q}$ defined by

$$
N_{q}(\mu)=\inf \left\{k>0: I_{q}(\mu / k) \leqq 1\right\}
$$

is a norm under which the space $A^{q}(v, Z)$ is a Banach space.

Proposition 1. The sets $K_{q}(v, Z)$ and $A^{q}(v, Z)$ are equal, and the respective norms satisfy the following inequality:

$$
N_{q}(\mu) \leqq\|\mu\|_{a} \leqq 2 N_{a}(\mu) \text { for all } \mu \in K_{q}(v, Z) \text {. }
$$

Proof. The proof of Lemma 7 of Rao [6, p. 563] applies here, with only the obvious changes needed.

It follows from Proposition 1 that the space $K_{q}(v, Z)$ is a Banach space.

Let $s \in S(V, Y)$ and $\mu \in K_{q, u}(v, Z)$. Define the integral $\int u(s, d \mu)$ by the formula

$$
\int u(s, d \mu)=\sum_{i} u\left(y_{i}, \mu\left(A_{i}\right)\right)
$$

where $s=\sum_{i} y_{i} c_{A_{i}}$ with $y_{i} \in Y$ and $\left\{A_{i}\right\}$ a finite family of disjoint sets from $V$. One can prove that $\int u(s, d \mu)$ is well defined and that the operator $\int u(\cdot, \cdot)$ is bilinear and continuous from the space $S(V, Y) \times K_{q, u}(v, Z)$ into the space $W$ and satisfies the relationship

$$
\left|\int u(s, d \mu)\right| \leqq\|s\|_{p, v}\|\mu\|_{q, u} .
$$

If we assume that the Young's function $p$ satisfies the $\Delta_{2}$-condition then from Th. 8 we may extend the operator $\int u(\cdot, \cdot)$ onto the space $L_{p}(v, Y) \times$ $K_{a, u}(v, Z)$. The extended operator is bilinear continuous on this space and 
satisfies the relationship

$$
\left|\int u(f, d \mu)\right| \leqq\|f\|_{p, v}\|\mu\|_{q, u} \quad \text { for } f \in L_{p}(v, Y), \mu \in K_{q, u}(v, Z) .
$$

3. Products of vector-valued set functions. For the volume spaces $\left(X_{i}, V_{i}, v_{i}\right)(i=1, \cdots, n)$ define the triple $(X, V, v)$ as follows: $X=$ $X_{1} \times \cdots \times X_{n}, V$ is the family of all sets of the form $A_{1} \times \cdots \times A_{n}$ where $A_{i} \in V_{i}$, and $v(A)=v_{1}\left(A_{1}\right) \cdots v_{n}\left(A_{n}\right)$ for $A \in V$. It is clear that $(X, V, v)$ is a volume space.

Let the volume space $(X, V, v)$ be the product of the volume spaces $\left(X_{i}, V_{i}, v_{i}\right)(i=1,2)$.

Theorem 2. Let $\mu \in K_{q}(v, Z)$ and $A \in V_{1}$ with $v_{1}(A)>0$. If we define the functions $\mu_{A}, v_{A}$ by the formulae

$$
\mu_{A}(B)=\mu(A \times B), \quad v_{A}(B)=v(A \times B) \quad \text { for all } B \in V_{2}
$$

then (i) $v_{A}$ is a volume on the prering $V_{2}$, (ii) the spaces $K_{q}\left(v_{A}, Z\right)$ and $K_{q}\left(v_{2}, Z\right)$ are equal, and (iii) $\mu_{A} \in K_{q}\left(v_{2}, Z\right)$.

Proof. Let $A \in V_{1}$. It is clear that $v_{A}$ is a volume on the prering $V_{2}$. Put $c=v_{1}(A)$ and assume $c>0$. That the sets $K_{q}\left(v_{A}, Z\right)$ and $K_{q}\left(v_{2}, Z\right)$ are equal will follow from Proposition 1 once we have shown that the sets $A^{q}\left(v_{A}, Z\right)$ and $A^{q}\left(v_{2}, Z\right)$ are equal.

Let $\mathscr{F}$ be a finite family of disjoint sets from the prering $V_{2}$. Let $\mu \in$ $A^{q}\left(v_{2}, Z\right)$, and let $k>0$ be such that $I_{q}(\mu / k)_{v_{2}} \leqq 1$. Then we have the following relations:

$$
\begin{aligned}
\sum\left\{q\left[c|\mu(B)| / k v_{A}(B)\right] v_{A}(B) / c: B \in \mathscr{F}\right\} & \\
& =\sum\left\{q\left[|\mu(B)| / k v_{2}(B)\right] v_{2}(B): B \in \mathscr{F}\right\} \leqq 1 .
\end{aligned}
$$

If $c>1$ then since $c q(x) \leqq q(c x)$ for all $x \geqq 0$ we have

If $c \leqq 1$ then

$$
\sum\left\{q\left[|\mu(B)| / k v_{A}(B)\right] v_{A}(B): B \in \mathscr{F}\right\} \leqq 1 .
$$

$$
\sum\left\{q\left[|\mu(B)| /(k / c) v_{A}(B)\right] v_{A}(B): B \in \mathscr{F}\right\} \leqq c \leqq 1 .
$$

Thus if $e=\min (c, 1)$ then $e N_{q}(\mu)_{v_{A}} \leqq N_{q}(\mu)_{v_{2}}$ and $\mu \in A^{q}\left(v_{A}, Z\right)$. Similarly one can show that $A^{q}\left(v_{A}, Z\right)$ is a subset of $A^{q}\left(v_{2}, Z\right)$ and that if $f=\max (c, 1)$ then $N_{q}(\mu)_{v_{2}} \leqq f N_{q}(\mu)_{v_{A}}$. To show that $\mu_{A} \in K_{q}\left(v_{2}, Z\right)$ we need only prove that $\mu_{A} \in A^{q}\left(v_{A}, Z\right)$, which follows from definitions, Proposition 1 and (ii) above.

THEOREM 3. Let $\mu \in K_{q, u}(v, Z)$ and $A \in V_{1}$. If the functions $\mu_{A}, v_{A}$ are defined as in Theorem 2 then $\mu_{A} \in K_{q, u}\left(v_{2}, Z\right)$ and $\left\|\mu_{A}\right\|_{q, u} \leqq\left(1+v_{1}(A)\right)\|\mu\|_{q, u}$ for all $A \in V_{1}$. 
Proof. It is clear that the function $\mu_{A}$ is finitely additive on the prering $V_{2}$ and that $\mu_{A}(B)=0$ when $v_{2}(B)=0$. Let $s=\sum_{i} y_{i} c_{A_{i}}$ be such that $\|s\|_{p, v_{2}} \leqq 1$. Then since $s^{\cdot} c_{A}$ is a simple function $s^{\cdot} c_{A} \in L_{p}(v, Y)$. From Young's inequality we have $\left\|s^{\cdot} c_{A}\right\|_{p, v} \leqq 1+\int p^{\circ}\left|s^{\cdot} c_{A}\right| d v$, and from Theorem 3 of Bogdanowicz [3] we have the relation $\int p^{\circ}\left|s^{\cdot} c_{A}\right| d v=$ $v_{1}(A) \int p \circ|s| d v_{2}$. From Th. 4 we have the estimate $\int p \circ|s| d v_{2} \leqq 1$, so that $\left\|s^{\cdot} c_{A}\right\|_{\mathcal{D}, v} \leqq a$, where $a=1+v_{1}(A)$. Thus we have

$$
\mid \sum_{i} u\left(y_{i} / a, \mu_{A}\left(A_{2 i}\right) \mid \leqq\|\mu\|_{q, u},\right.
$$

so that $\left\|\mu_{A}\right\|_{q, u} \leqq a\|\mu\|_{q, u}$ and $\mu_{A} \in K_{q, u}\left(v_{2}, Z\right)$.

We remark that Theorem 2 could also be proved in a manner analogous to the proof of Theorem 3, the proof of which would yield an inequality similar to that of Theorem 3.

Let $Z_{i}(i=1, \cdots, n), W$ be Banach spaces and let $u$ be an $n$-linear continuous operator from the product of the spaces $Z_{1}, \cdots, Z_{n}$ into the space $W$. Let $B\left(Z_{1}, \cdots, Z_{n} ; W\right)$ denote the space of all such operators $u$. Denote the norms in the above spaces by $|\cdot|$. Let $(X, V, v)$ be the product volume space formed from the volume spaces $\left(X_{i}, V_{i}, v_{i}\right)(i=1, \cdots, n)$.

THEOREM 4. Assume that the Young's function $q$ satisfies the $\Delta^{\prime}$ condition. If $\mu_{i} \in K_{q}\left(v_{i}, Z_{i}\right)$ for $i=1, \cdots, n$ then $\mu \in K_{q}(v, W)$, where

$$
\mu\left(A_{1} \times \cdots \times A_{n}\right)=u\left(\mu_{1}\left(A_{1}\right), \cdots, \mu_{n}\left(A_{n}\right)\right) \text { for } A \in V .
$$

Proof. The proof is given in [9] for Orlicz spaces of finitely additive set functions generated from a charge space. The proof there is applicable here.

4. A Fubini theorem for the Orlicz space $L_{p}(v, Y)$. Let $(X, V, v)$ be the product volume space of the volume spaces $\left(X_{i}, V_{i}, v_{i}\right)$ for $i=1,2$. Let $Y, Z_{1}, Z_{2}, W$ be Banach spaces. Assume that $w \in B\left(Y, Z_{1}, Z_{2} ; W\right)$ and define the operator $w_{1}$ by $w_{1}\left(y, z_{2}\right)=w\left(y, \cdot, z_{2}\right)$ for $y \in Y, z_{2} \in Z_{2}$. It is clear that $w_{1} \in B\left(Y, Z_{2} ; Y_{1}\right)$, where $Y_{1}=B\left(Z_{1} ; W\right)$. Define $w_{0}\left(y_{1}, z_{1}\right)=y_{1}\left(z_{1}\right)$ for $y_{1} \in Y_{1}, z_{1} \in Z_{1}$ and notice that $w_{0} \in B\left(Y_{1}, Z_{1} ; W\right)$ and $|w|=\left|w_{1}\right|$ and $\left|w_{0}\right|=1$.

Let $N\left(v_{1}, Y_{1}\right)$ denote the subset of those functions from the space $L_{p}\left(v_{1}, Y_{1}\right)$ whose seminorms are zero. The space $N\left(v_{1}, Y_{1}\right)$ is a linear space and the quotient space $L_{p}\left(V_{1}, Y_{1}\right) / N\left(v_{1}, Y_{1}\right)$ is a Banach space with norm $\|\cdot\|_{p, v_{1}}$ defined by the formula

$$
\|[f]\|_{p, v_{1}}=\|f\|_{\mathcal{D}, v_{1}} \text { for all } f \in L_{p}\left(v_{1}, Y_{1}\right)
$$


Let us assume that the Young's functions $p, q$ satisfy respectively the $\Delta_{2}$-condition and the $\Delta^{\prime}$-condition, with $k$ the associated $\Delta^{\prime}$-constant.

THEOREM 5. Let $f \in L_{p}(v, Y)$ and $\mu_{2} \in K_{q}\left(v_{2}, Z_{2}\right)$. Then the following statements are true:

(i) There exists a $v_{1}$-null set $D$ such that $f\left(x_{1}, \cdot\right) \in L_{p}\left(v_{2}, Y\right)$ if $x_{1} \notin D$.

(ii) If the function $t$ is defined by the formula $t\left(x_{1}\right)=\left\|f\left(x_{1}, \cdot\right)\right\|_{p, v}$ if $x_{1} \notin D$ and 0 otherwise then $t \in L_{p}\left(v_{1}, R\right)$ and $t$ satisfies the inequality $\|t\|_{D_{. v_{1}}} \leqq$ $\int p \circ|f| d v+k$.

(iii) The operator $\bar{f}=r\left(f, \mu_{2}\right)$ defined by the formula

$$
f\left(x_{1}\right)=\int w_{1}\left(f\left(x_{1}, x_{2}\right), \mu_{2}\left(d x_{2}\right)\right)
$$

if $x_{1} \notin D$ is bilinear from the space $L_{p}(v, Y) \times K_{q}\left(v_{2}, Z_{2}\right)$ into the space $L_{p}\left(v_{1}, Y_{1}\right) / N\left(v_{1}, Y_{1}\right)$ and satisfies the inequality $\|\widetilde{f}\|_{p_{, v_{1}}} \leqq\left\|\mu_{2}\right\|_{Q}\|t\|_{p . v_{1}}|w|$.

(iv) $\int w\left(f, d \mu_{1}, d \mu_{2}\right)=\int w_{0}\left(r\left(f, \mu_{2}\right), d \mu_{1}\right)$ for all $f \in L_{p}(v, Y), \mu_{i} \in K_{q}\left(v_{i}, Z_{i}\right)$ for $i=1,2$.

Proof. Let $f \in L_{p}(v, Y)$. By Th. 1 and Tonelli's theorem there is a $v_{1-}$ null set $D_{1}$ such that $f\left(x_{1}, \cdot\right) \in M\left(v_{2}, Y\right)$ if $x_{1} \notin D_{1}$. By the corollary to Th. 4 and by Theorem 3 of [3] there is a $v_{1}$-null set $D_{2}$ such that $p \circ\left|f\left(x_{1}, \cdot\right)\right| \epsilon$ $L\left(v_{2}, R\right)$ if $x_{1} \notin D_{2}$. Put $D=D_{1} \cup D_{2}$ and notice that $D$ is a $v$-null set. Let $s \in S\left(V_{2}, R\right)$ be such that $\|s\|_{p, v_{2}} \leqq 1$ and assume that $x_{1} \notin D$. Then we get the estimate

so that

$$
\int\left|f\left(x_{1}, x_{2}\right)\right| s\left(x_{2}\right) v_{2}\left(d x_{2}\right) \leqq \int p\left(\left|f\left(x_{1}, x_{2}\right)\right|\right) v_{2}\left(d x_{2}\right)+1,
$$

$$
\left\|f\left(x_{1}, \cdot\right)\right\|_{p, v_{2}} \leqq \int p\left(\left|f\left(x_{1}, x_{2}\right)\right|\right) v_{2}\left(d x_{2}\right)+1 .
$$

It follows from Th. 1 that $f\left(x_{1}, \cdot\right) \in L_{p}\left(v_{2}, Y\right)$ if $x_{1} \notin D$.

For the operator $\bar{f}$, as defined in the theorem statement, put $\bar{f}\left(x_{1}\right)=$ $0 \in Y_{1}$ if $x_{1} \in D$. We shall first show that the function $\bar{f} \in M\left(v_{1}, Y_{1}\right)$.

Define the function $b_{m}$ by the formula

$$
\begin{aligned}
b_{m}(y) & =y & & \text { if }|y| \leqq m, \\
& =(m /|y|) y & & \text { if }|y|>m,
\end{aligned}
$$

for all $y \in Y$ and for $m=1,2, \cdots$.

Since $f \in M(v, Y)$ there is a sequence of functions $r_{n} \in S(V, Y)$ and a $v$-null set $A$ such that $r_{n}(x) \rightarrow f(x)$ if $x \notin A$. Let $C$ be the $v$-null set such that the section $A\left(x_{1}\right)=\left\{x_{2}:\left(x_{1}, x_{2}\right) \in A\right\}$ is a $v_{2}$-null set if $x_{1} \notin C$. Let the set $A_{n}$ be the support of the function $r_{n}$ and put $B_{n}=A_{1} \cup \cdots \cup A_{n}$, for $n=1,2, \cdots$. 
If for an appropriate strictly increasing sequence of positive integers $m(n)$ we define the function $s_{n}$ by the formula $s_{n}(x)=c_{B_{n}}(x) b_{n}\left(r_{m(n)}(x)\right)$ for all $x \in X$, for $n=1,2, \cdots$, then we get $\left\|f\left(x_{1}, \cdot\right)-s_{n}\left(x_{1}, \cdot\right)\right\|_{p, v_{2}} \rightarrow 0$ if $x_{1} \notin D \cup C$. Define the function $\bar{s}_{n}$ by the formula $\bar{s}_{n}\left(x_{1}\right)=\int w_{1}\left(s_{n}\left(x_{1}, x_{2}\right), \mu_{2}\left(d x_{2}\right)\right)$ for all $x_{1} \in X_{1}$, for $n=1,2, \cdots$. We have the estimate

$$
\left|\bar{s}_{n}\left(x_{1}\right)-\bar{f}\left(x_{1}\right)\right| \leqq\left\|s_{n}\left(x_{1}, \cdot\right)-f\left(x_{1}, \cdot\right)\right\|_{p, v_{2}}\left\|\mu_{2}\right\|_{Q}|w|
$$

if $x_{1} \notin D \cup C$. Thus $\bar{s}_{n} \rightarrow \bar{f} v_{1}$-a.e., and since $\bar{s}_{n} \in S\left(V_{1}, Y_{1}\right)$ it follows from Theorem 1 of [2] that $\bar{f} \in M\left(v_{1}, Y_{1}\right)$.

Let $s \in Q\left(v_{2}, R^{+}\right)$and $s_{0} \in Q\left(v_{1}, R^{+}\right)$. From Young's inequality and the fact that $q$ satisfies the $\Delta^{\prime}$-condition we get the following inequality:

$$
\int\left|f\left(x_{1}, x_{2}\right)\right| s\left(x_{2}\right) v_{2}\left(d x_{2}\right) s_{0}\left(x_{1}\right) \leqq \int p\left(\left|f\left(x_{1}, x_{2}\right)\right|\right) v_{2}\left(d x_{2}\right)+k q\left(s_{0}\left(x_{1}\right)\right) v_{1} \text {-a.e. }
$$

If we define the function $t$ on $X_{1}$ by $t\left(x_{1}\right)=\left\|f\left(x_{1}, \cdot\right)\right\|_{p, v_{2}}$ if $x_{1} \notin D$, and 0 otherwise then we get the estimates

and

$$
\left|\bar{f}\left(x_{1}\right)\right| s_{0}\left(x_{1}\right) \leqq t\left(x_{1}\right)\left\|\mu_{2}\right\|_{q}|w| s_{0}\left(x_{1}\right)
$$

$$
t\left(x_{1}\right) s_{0}\left(x_{1}\right) \leqq \int p\left(\left|f\left(x_{1}, x_{2}\right)\right|\right) v_{2}\left(d x_{2}\right)+k q\left(s_{0}\left(x_{1}\right)\right) \quad v_{1} \text {-a.e. }
$$

If we temporarily assume that the function $t$ is $v_{1}$-measurable then by integrating the last two inequalities and by taking the appropriate suprema we have the following two inequalities:

$$
\|\bar{f}\|_{p, v_{1}} \leqq\|t\|_{p, v_{1}}\left\|\mu_{2}\right\|_{q}|w|, \quad\|t\|_{p, v_{1}} \leqq \int p \circ|f| d v+k .
$$

Thus $\bar{f} \in L_{p}\left(v_{1}, Y_{1}\right) / N\left(v_{1}, Y_{1}\right)$. The bilinearity of the operator $r$ is evident.

We next show that the function $t$ is locally $v_{1}$-summable and that $t$ has a $\left(V_{1}\right)_{\sigma}$ support. The latter is true because $f \in L_{p}(v, Y)$. Fix $B \in V_{1}$ for the remainder of this discussion and choose $s \in Q\left(v_{2}, R^{+}\right)$. Notice that $\left|f\left(x_{1}, \cdot\right)\right| s(\cdot) \in L\left(v_{2}, R\right)$ if $x_{1} \notin D$. If we put $g_{s}\left(x_{1}\right)=c_{B}\left(x_{1}\right) \int\left|f\left(x_{1}, x_{2}\right)\right| s\left(x_{2}\right) v_{2}\left(d x_{2}\right)$ if $x_{1} \notin D$ and 0 otherwise then from Theorem 3 of [3] we have that $g_{s} \in$ $L\left(v_{1}, R\right)$. Define the function $h$ by the formula

$$
h\left(x_{1}\right)=c_{B}\left(x_{1}\right)\left[1+\int p \circ\left|f\left(x_{1}, x_{2}\right)\right| v_{2}\left(d x_{2}\right)\right]
$$

if $x_{1} \notin D$ and 0 otherwise, where $p \circ\left|f\left(x_{1}, \cdot\right)\right|$ is $v_{2}$-summable by Th. 4 . From Theorem 3 of [3] we have that $h \in L\left(v_{1}, R\right)$. From Young's inequality we have $g_{s}\left(x_{1}\right) \leqq h\left(x_{1}\right)$ for all $x_{1}$. Let $G=\left\{g_{s}: s \in Q\left(v_{2}, R^{+}\right)\right\}$then $G$ is a bounded set from $L\left(v_{1}, R\right)$. Since $L\left(v_{1}, R\right)$ is order complete with respect to pointwise domination we have that the function $t c_{B}=\sup \left\{g_{s}: s \in Q\left(v_{2}, R^{+}\right)\right\}$ is $v_{1}$-summable. 
Part (iv) of the theorem can easily be shown true for simple functions. That it is true for functions from $L_{p}(v, Y)$ follows from the density of simple functions in $L_{p}(v, Y)$ (Th. 8) and from the boundedness and coordinate-wise linearity of the integral.

REMARK. The statement derived from Theorem 5, excluding part (iv), by replacing the space $K_{q}\left(v_{2}, Z_{2}\right)$ by the space $K_{q . w}\left(v_{2}, Z_{2}\right)$ and by replacing the value $\left\|\mu_{2}\right\|_{q}|w|$ by the value $\left\|\mu_{2}\right\|_{a, w}$ is true. The proof is similar to that of Theorem 5 .

\section{REFERENCES}

1. W. M. Bogdanowicz, A generalization of the Lebesgue-Bochner-Stieltjes integral and a new approach to the theory of integration, Proc. Nat. Acad. Sci. U.S.A. 53 (1965), 492-498. MR 31 \#306.

2. - An approach to the theory of Lebesgue-Bochner measurable functions and to the theory of measure, Math. Ann. 164 (1966), 251-269. MR 34 \#1483.

3. - Fubini theorems for generalized Lebesgue-Bochner-Stieltjes integral, Proc. Japan Acad. 41 (1965), 979-983. MR 34 \#1484.

4. J. Diestel, An approach to the theory of Orlicz spaces of Lebesgue-Bochner measurable functions, Math. Ann. 186 (1970), 20-33. MR 41 \#7417.

5. M. A. Krasnosel'skiǐ and Ja. B. Rutickiĭ, Convex functions and Orlicz spaces, GITTL, Moscow, 1958; English transl., Noordhoff, Groningen, 1961. MR 21 \#5144; MR 23 \#A4016.

6. M. M. Rao, Linear functionals on Orlicz spaces: general theory, Pacific J. Math. 25 (1968), 553-585.

7. J. J. Uhl, Orlicz spaces of finitely additive set functions, Studia Math. 29 (1967), 19-58. MR 37 \#1985.

8. V. Zander, Fubini theorem for Orlicz spaces of Lebesgue-Bochner measurable functions, Notices Amer. Math. Soc. 17 (1970), 768. Abstract \#677-28-2.

9. - Products of finitely additive set functions from Orlicz spaces, Pacific J. Math. 35 (1970), 799-804.

Department of Mathematics, West Georgia College, Carrollton, Georgia 30117 